\title{
Oral contraceptives do not appear to affect cystic fibrosis disease severity
}

\author{
Natalie G. Kernan*, Eric W.F.W. Alton*, Paul Cullinan ${ }^{\#,+}$, Uta Griesenbach ${ }^{\star,+}$ \\ and Diana Bilton ${ }^{\uparrow}+$
}

ABSTRACT: Several studies suggest that sex may affect cystic fibrosis (CF) disease severity, with females with CF being more severely affected. In this context, it has been suggested that sex hormones may influence the CF phenotype. A large proportion of females with CF regularly use oral contraceptives (OCs), but the effect of their use on disease severity is unclear. Here, we retrospectively assessed the effects of OCs on clinical outcomes in females with CF.

Data from 681 females were available, of whom $42 \%$ had taken OCs for varying periods of time. We first performed an inter-patient analysis comparing annual change in \% predicted forced expiratory volume in $1 \mathrm{~s}$, body mass index and total days of intravenous antibiotic use over a 5-yr study period in 57 females exposed to and 57 females not exposed to OCs. There were no differences between the two groups. We next performed an intra-patient analysis of the same outcomes over a 3-yr period of $\mathrm{OC}$ exposure and a 3-yr period of no $\mathrm{OC}$ exposure in the same patient (exposure followed by non-exposure, $n=27$; non-exposure followed by exposure, $n=23$ ), but again did not detect any differences in any of the clinical outcomes.

Our data suggests that the use of OCs does not affect CF disease severity.

KEYWORDS: Cystic fibrosis transmembrane conductance regulator, hormones, lung disease, oestrogen

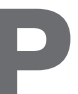
ulmonary disease progression in cystic fibrosis (CF) is heterogeneous. This may in part be related to the different classes of cystic fibrosis transmembrane conductance regulator (CFTR) mutations having different effects on protein function [1]. However, even patients with the same mutation frequently differ with respect to pulmonary disease progression. It has been shown that socio-economic status, adherence and access to treatment as well as modifier genes contribute to the progression of CF lung disease [2]. In addition, it has been suggested that sex may have an effect on CF mortality, with females dying earlier than males [3-5], and on morbidity, since it has been suggested that females with CF acquire chronic Pseudomonas aeruginosa infection earlier and that this is linked to an accelerated decline of pulmonary function [6, 7]. VERMA et al. [8] suggest that the sex gap in childhood and adolescence closes when both sexes receive standardised aggressive treatments in a single centre.

It has been suggested that sex hormones may directly influence the CF phenotype by altering ion transport in epithelial cells. These effects have been investigated in vivo by SWEEZEY et al. [9], who showed that the amiloride-insensitive component of nasal potential difference (NPD) varies during the menstrual cycle in $\mathrm{CF}$ females. During the luteal (high progesterone) phase, amiloride-insensitive NPD was significantly higher than during the follicular (low progesterone) phase. This suggests that elevated progesterone during the luteal phase may alter ion transport across the respiratory epithelium, which may alter the height of the periciliary liquid and, therefore, mucociliary clearance.

In vitro studies assessing the effects of sex hormones on ion transport have produced conflicting results, with some studies reporting inhibition of chloride transport $[10,11]$ and others showing increased CFTR transcription after sex hormone exposure [12-14]. FANELLi et al. [15] observed an increase in the processing of misfolded CFTR leading to improved ion transport in a CF human bronchiolar epithelial cell line, and proposed that oestrogens rescue misfolded $\Delta \mathrm{F} 508$ CFTR from proteosomal degradation, thereby increasing the amount of functional CFTR at the cell membrane. In addition to direct effects on ion transport and CFTR expression and maturation, CHOTIRMALL et al. [16] have recently shown that oestrogens inhibit interleukin (IL)-8 secretion via upregulation of the secretory leukoprotease inhibitor (SLPI) in an immortalised CF cell line. This
AFFILIATIONS

*Dept of Gene Therapy, Imperial

College London,

${ }^{*}$ Dept of Occupational and Environmental Medicine, Royal Brompton Hospital, and

"Cystic Fibrosis Centre, Royal Brompton Hospital, London, UK. ${ }^{+}$These authors contributed equally.

CORRESPONDENCE

U. Griesenbach

Dept of Gene Therapy National Heart and Lung Institute Imperial College London Manresa Road

London

SW3 6LR

UK

E-mail: u.griesenbach@

imperial.ac.uk

Received:

Jan 302012

Accepted after revision:

April 012012

First published online:

May 032012 
pathway may directly affect the host response during highoestrogen phases of the menstrual cycle. Sex hormones have also been implicated in regulating immune and inflammatory responses in various pre-clinical and clinical models [17].

Combined, these results imply that sex hormones may have an effect on CF lung disease. Only one short-term study of 12 females assessed lung function in relation to sex hormone levels, showing that lung function (as measured by forced expiratory volume in $1 \mathrm{~s}(\mathrm{FEV} 1)$ ) changed in relation to the menstrual cycle. FEV1 was significantly higher during the luteal phase (high levels of oestrogen and progesterone) compared with ovulation (high oestrogen and low progesterone) and menstruation (low oestrogen and progesterone) [18].

As the median age of survival increases, more females with $\mathrm{CF}$ reach child-bearing age and use oral contraceptives (OCs) [19, 20]. The use of OCs by females with CF provides a clinical model in which to test the hypothesis that exogenous oestrogen and/or progesterone may have a significant clinical effect on the course of the disease. Here, we performed a retrospective inter- and intra-patient comparison study to assess whether OC use affects disease severity in $\mathrm{CF}$ as measured by annual change in pulmonary function (\% predicted FEV1), body mass index (BMI) and the need for intravenous antibiotics.

\section{METHODS AND MATERIALS \\ Database}

We used information collected at annual review, during a period of clinical stability, for patients under the care of the Adult Cystic Fibrosis Unit of Royal Brompton Hospital, London, UK. Information on the use of OCs was available for the years 1981-2010 and recorded in each year as "yes" or "no", or as "unknown" if the information was missing. Females were excluded from analysis if they had unknown OC use for all years of follow-up available in the database. We also extracted annual information on FEV1\% pred, BMI and i.v. antibiotic use over the previous year (total days of treatment). Data was taken from the Royal Brompton and Harefield Trust CF database. Ethics committee (Royal Brompton, Harefield and NHLI Research Ethics Committee) approval has been granted and all patients on the database gave signed consent for anonymous data to be used for research purposes.

\section{Inter-patient cohort comparison}

We identified all females who had used OCs for a minimum of 4 out of 5 yrs during a continuous 5-yr period. Females in this "exposed" cohort were matched by age to a female who, during the same 5-yr period, had never used OCs ("not exposed"). Information on FEV1 \% pred, BMI and i.v. antibiotic use was extracted for each year of the relevant 5-yr period; annual changes in FEV1 \% pred and BMI were estimated using linear regression. Days of i.v. antibiotic use during each period were summed. To confirm that the two cohorts were similar at the start of analysis we compared FEV1 \% pred, BMI and days on i.v. antibiotics at the start of analysis.

\section{Intra-patient comparison}

In addition to the inter-patient comparison, we also decided $a$ priori to perform an intra-patient comparison. We identified females $(n=27)$ who used OCs for three consecutive years ("exposed" period) followed by 3 yrs of non-use ("not exposed" period); and, conversely and separately, females who did not take OCs for three consecutive years followed by 3 years of continuous use $(n=23)$. As above, annual changes in FEV1 \% pred and BMI were estimated for each exposed and not exposed period using linear regression, and days of $i . v$. antibiotic use during each period were summed. Data obtained for the exposed and not exposed period were then compared for each female.

\section{Statistical analysis}

Statistical analysis was performed using the GraphPad PRISM 4.0 software package (GraphPad Software Inc., San Diego, CA, USA). After assessment of normal distribution, parametric data were analysed using an unpaired or paired t-test, as appropriate. Non-parametric data were analysed using the MannWhitney U-test and the Wilcoxon matched pairs test for unpaired data and paired data, respectively. The null hypothesis was rejected at $\mathrm{p}<0.05$.

\section{RESULTS \\ Patient demographics}

In the period from 1981 to $2010,42 \%$ of females on the database ( $n=286$ females aged 16-49 yrs) reported use of OCs on at least one annual review. $43 \%(n=290)$ had never used OCs and $15 \%$ $(n=104)$ had never responded either "yes" or "no" and were

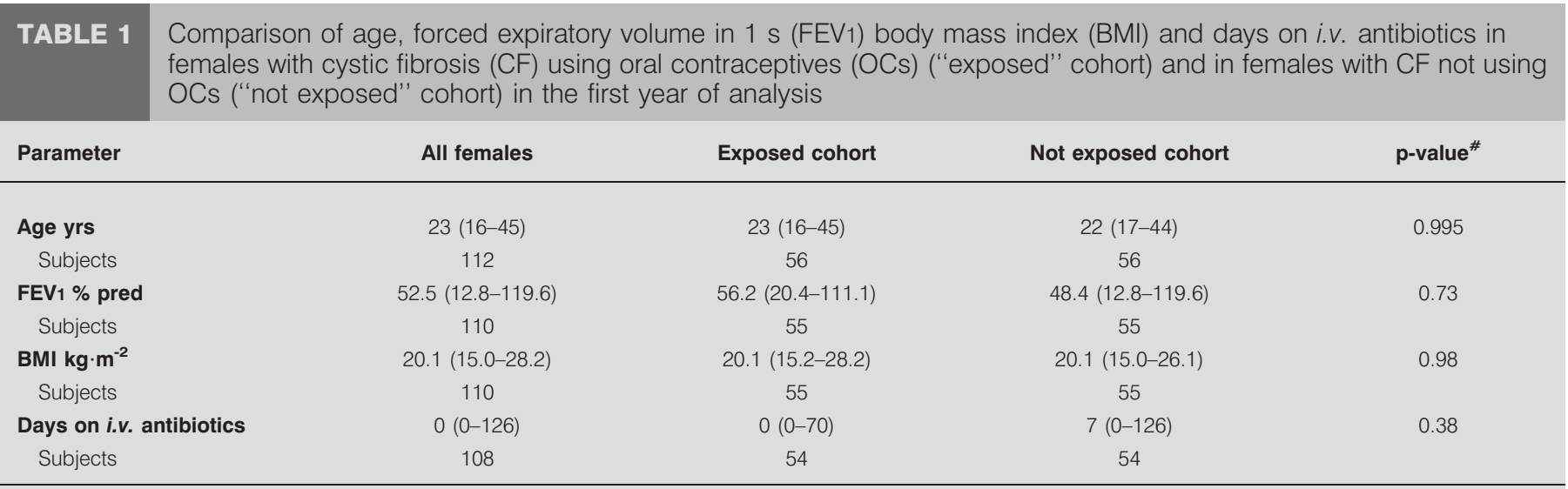

Data are presented as median (range) or $\mathrm{n}$, unless otherwise stated. \% pred: \% predicted. *: p-values relate to comparisons between females exposed and not exposed to OCs. 


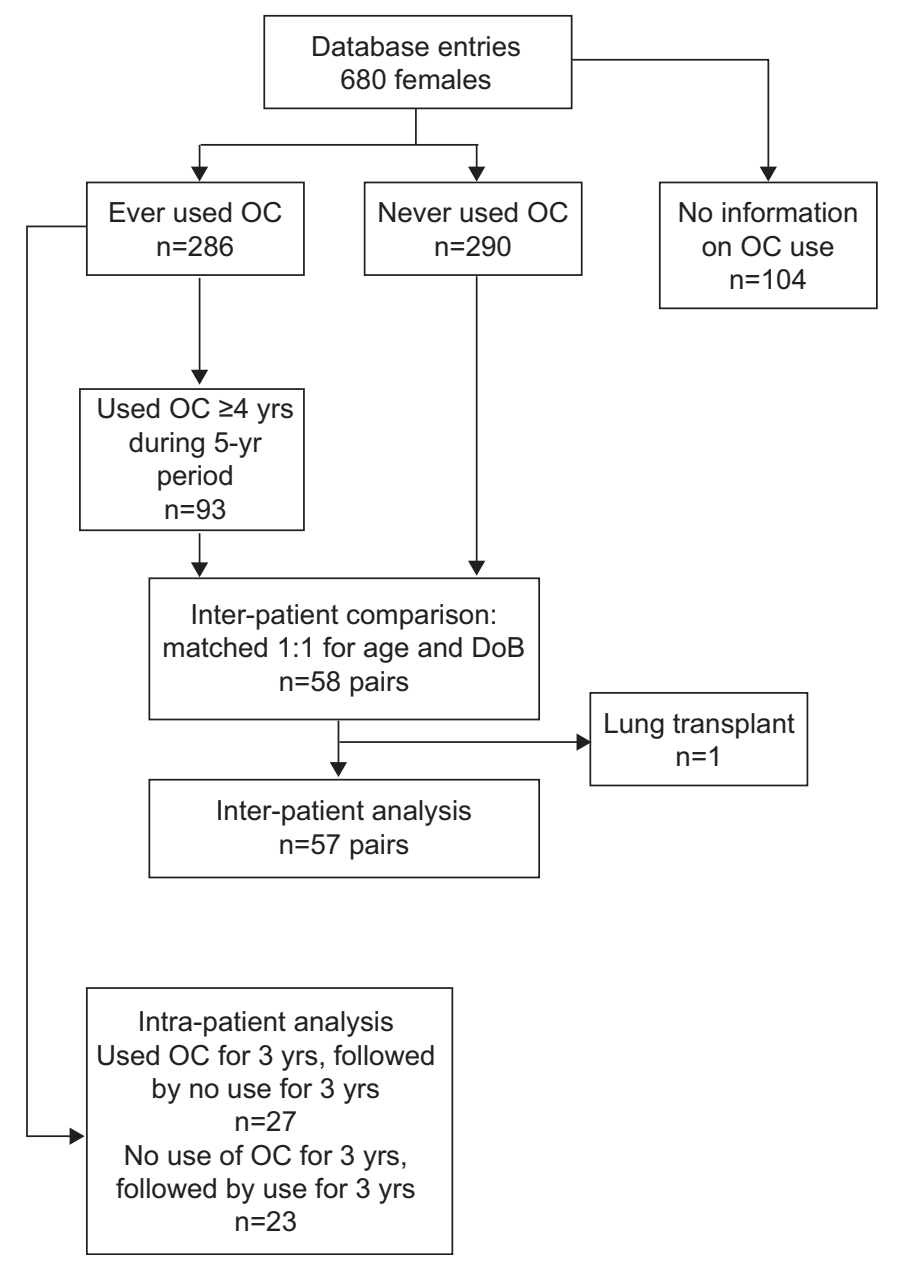

FIGURE 1. Flow chart of patient selection for "exposed" and "not exposed" cohorts for inter- and intra-patient comparison. OC: oral contraceptive; DoB: date of birth.

therefore excluded from subsequent consideration (table 1). Years of OC use ranged from $1(n=101)$ to 21 yrs $(n=1)$, but $\sim 33 \%(n=93)$ of females had continuously used OCs for 4 5 yrs. This frequency dropped to $\sim 10 \%(n=31)$ for females who had continuously used OCs for 6-7 yrs.

\section{Inter-patient cohort comparison}

Females were assigned to the exposed and not exposed cohorts as outlined in figure 1.93 were identified for the exposed cohort; the database was then screened for "matching" females who had never taken OCs (not exposed cohort) and 57 pairs were identified. However, incomplete data for the study period led to the subsequent exclusion of a further two (for FEV1 \% pred and BMI analysis) or three (analysis of i.v. antibiotic use) matched pairs.

Comparison of subjects in the exposed and non-exposed groups at the start of the analysis period (year 1) showed that they were of similar age. Females who had not taken OCs had a lower median FEV1 \% pred and more days on i.v. antibiotics, but this did not reach significance (table 1).

There were no differences between the two cohorts in annual change in FEV1 \% pred during the period of analysis $(n=55$ per group; exposed, $-1.87 \%$ (-11.5-10.4\%); not exposed, $-1.03 \%$
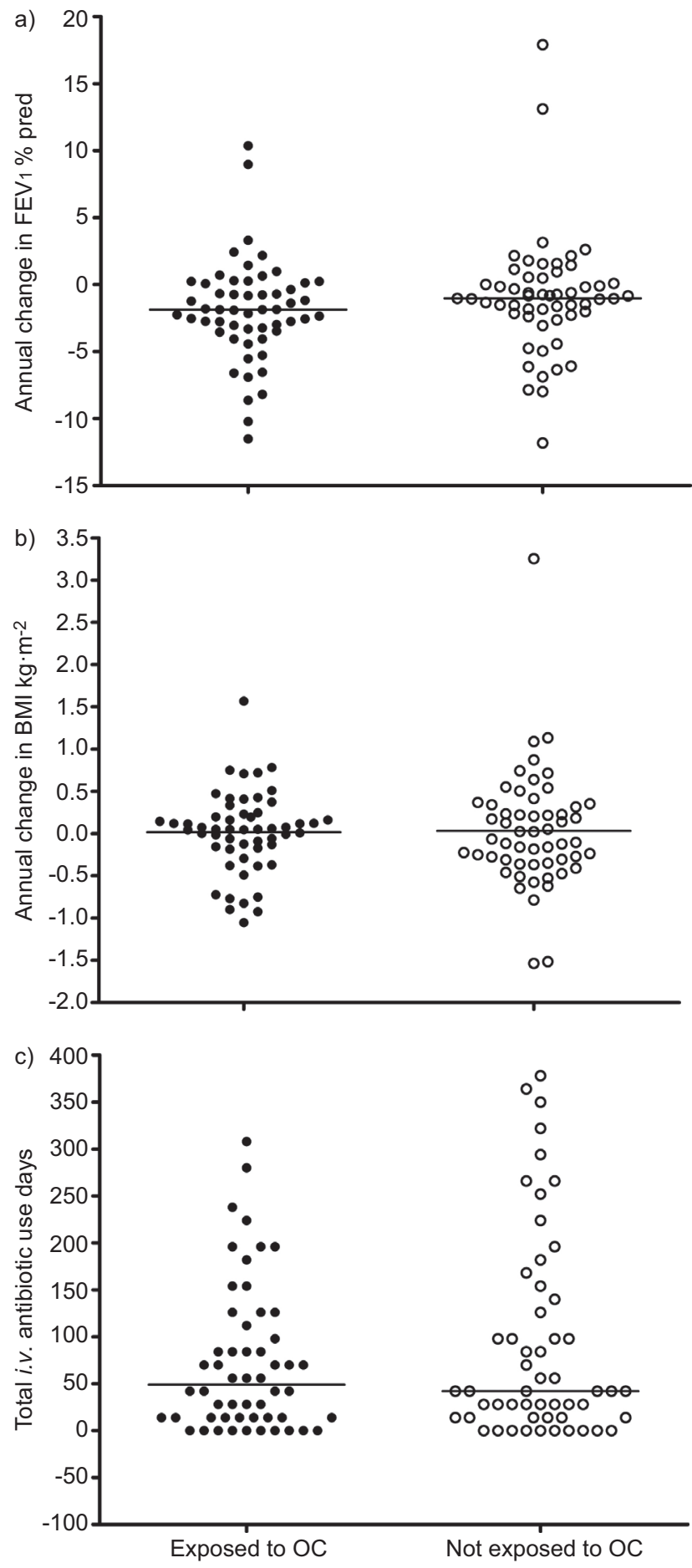

FIGURE 2. Comparison of disease severity during the 5-yr study period in females with cystic fibrosis (CF) using oral contraceptives (OCs) ("exposed" cohort) and females with CF not using OCs ("not exposed" cohort). a) Annual change in forced expiratory volume in $1 \mathrm{~s}$ (FEV1) \% predicted (\% pred); b) annual change in body mass index (BMI); c) total number of days of i.v. antibiotic use during the 5-yr study period. Each symbol represents one individual. Parametric and non-parametric statistical analysis was performed as appropriate. Horizontal bars indicate group mean (b) or median ( $a$ and $\mathrm{c}$ ) as appropriate for parametric or non-parametric data. 


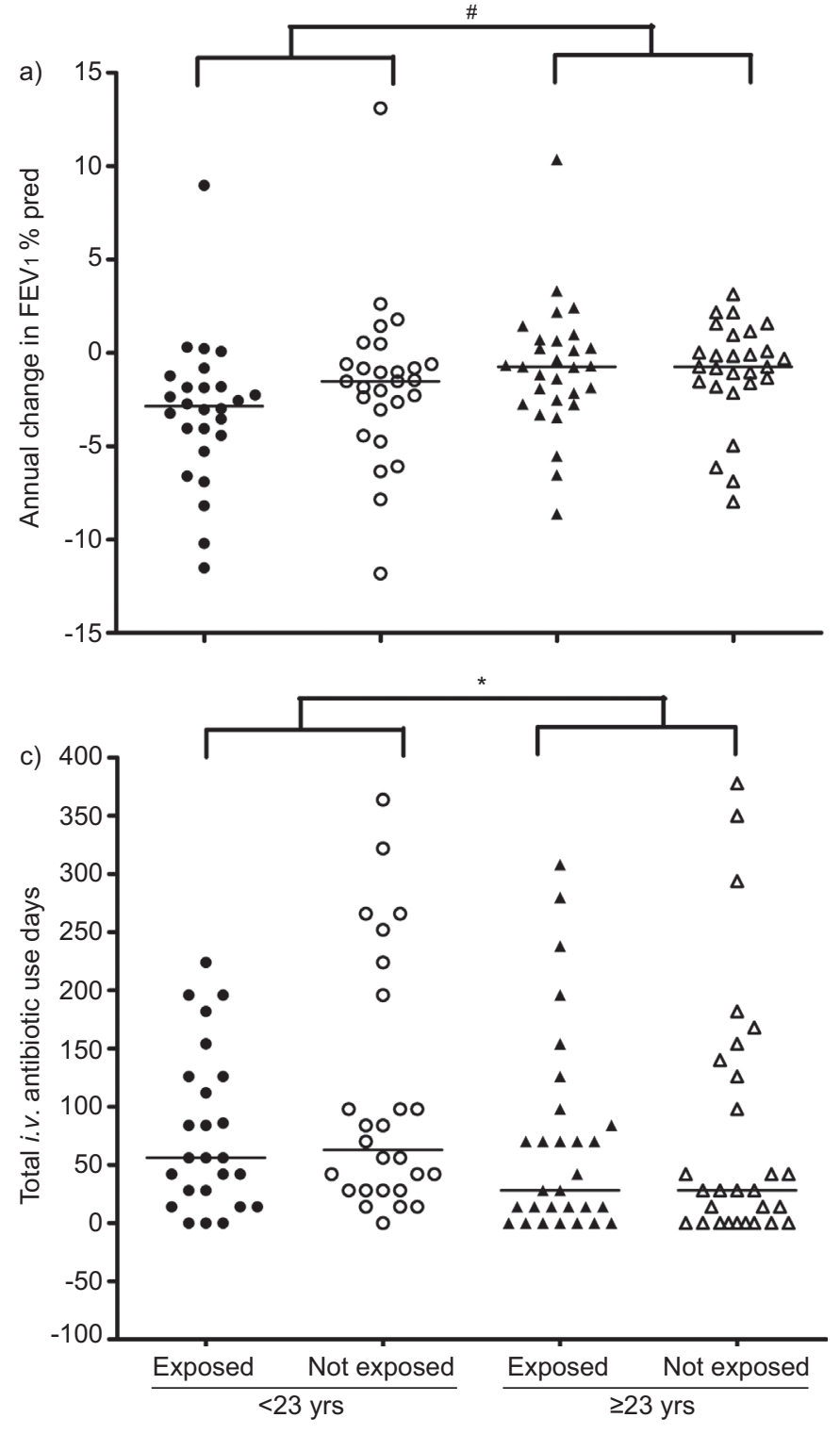

(-11.9-17.9\%); $\mathrm{p}=0.115)$ (fig. $2 \mathrm{a}$ ); in annual change in BMI $\left(\mathrm{n}=55\right.$ per group; exposed $0.05(-1.05-1.57) \mathrm{kg} \cdot \mathrm{m}^{-2}$; not exposed, $\left.-0.07(-1.54-3.25) \mathrm{kg} \cdot \mathrm{m}^{-2} ; \mathrm{p}=0.891\right)$ (fig. 2b) or in total days of i.v. antibiotics ( $\mathrm{n}=54$ per group; exposed, $49(0$ 308) days; not exposed, 42 (0-378) days; $\mathrm{p}=0.685)$ (fig. 2c ).

Subgroup analysis using less than or greater than or equal to the median age of 23 yrs was also performed. Within each age group there was no difference in annual change in FEV1 \% pred $(<23$ yrs, $\mathrm{p}=0.062 ; \geqslant 23$ yrs, $\mathrm{p}=0.762)$, BMI $(<23$ yrs, $\mathrm{p}=0.869$; $\geqslant 23$ yrs, $\mathrm{p}=0.876)$ or total days of i.v. antibiotics $(<23 \mathrm{yrs}$ $p=0.468 ; \geqslant 23$ yrs $p=0.860$ ) (fig. $3 a-c$ and table 2 ). When all subjects $<23$ yrs of age at the start of analysis were compared to all subjects $\geqslant 23$ yrs of age at the start of analysis there was a significant difference between the groups for all three parameters during the 5-yr period (annual change in FEV1 \% pred, $\mathrm{p}<0.005$; annual change in BMI, $\mathrm{p}<0.05$; total days of i.v. antibiotics, $\mathrm{p}<0.05$ ) (fig. $3 \mathrm{a}-\mathrm{c}$ and table 2), indicating that younger females with CF have a steeper rate of decline in FEV1 and BMI, and a greater i.v. antibiotic usage than older females.

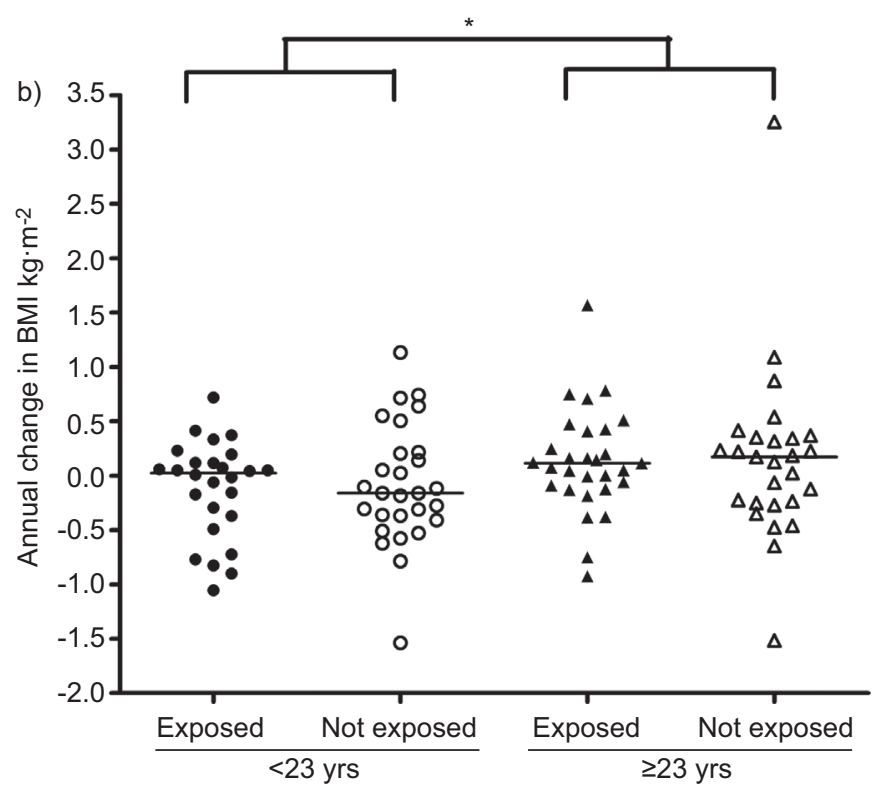

FIGURE 3. Subgroup analysis comparing disease severity in young and older females with cystic fibrosis (CF) who used oral contraceptives (OCs) ("exposed" cohort) and females with CF who did not use OCs ("not exposed" cohort). Females were grouped by age ( $<23$ and $\geqslant 23 \mathrm{yrs}$ ), and disease severity in exposed and not exposed cohorts was compared. a) Annual change in forced expiratory volume in $1 \mathrm{~s}$ (FEV1) \% predicted (\% pred); b) annual change in body mass index (BMI); c) total number of days of i.v. antibiotic use during the $5-y r$ study period. Each symbol represents one individual. Parametric and nonparametric statistical analysis was performed as appropriate. Horizontal bar indicates group mean (b) or median ( $a, c)$ as appropriate for parametric or non-parametric data. ${ }^{*}: \mathrm{p}<0.05 ;{ }^{*}: \mathrm{p}<0.005$ when comparing younger and older subjects.

\section{Intra-patient cohort comparison}

We next assessed annual change in FEV1 \% pred and BMI and total days on i.v. antibiotics over a period of OC use (exposed period) and a period of no OC use (not exposed period) in the same female (fig. 1). There were no differences in any of the parameters when comparing periods of OC use to periods of no OC use regardless of order (table 3).

\section{DISCUSSION}

The literature assessing the effects of sex hormones on ion transport in airway epithelial cells is conflicting, and studies proposing potential beneficial and detrimental effects of oestrogen on ion transport in CF have been published. Here, we conducted a retrospective study aimed at assessing the effect of OC use in females with CF. Our data suggest that OC use does not affect CF disease severity.

As the median age of survival increases, more females with $\mathrm{CF}$ reach child-bearing age and use OCs $[19,20]$. In our centre, $\sim 43 \%$ of females with CF reported OC use at least once when 


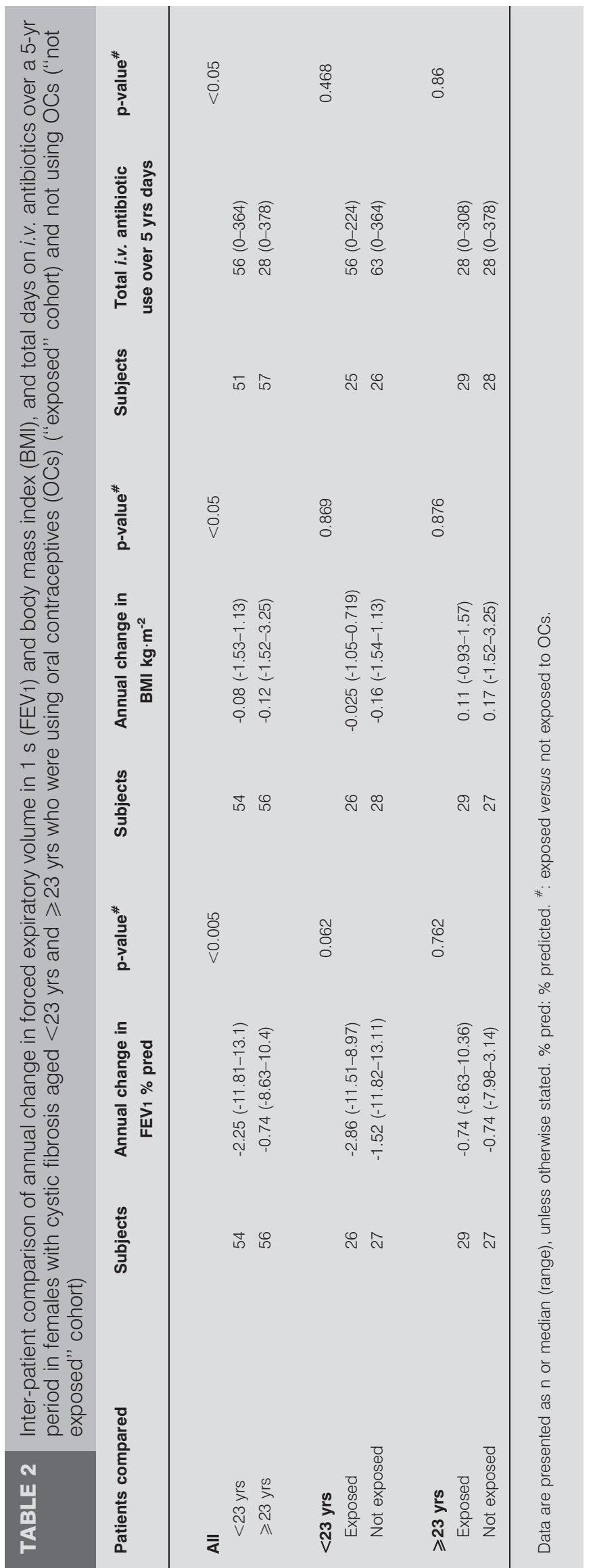

questioned during annual follow-up, a percentage similar to frequencies reported by CONWAY et al. [21]. However, the effects of OCs on disease severity have not been extensively studied. We are aware of only one comparatively small and short study, which assessed the effect of OCs on lung function. FitZPATRICK et al. [19] studied 12 females using OCs and reported that lung function did not decline over a 6-month period. Our study was conducted over 5 yrs of follow-up, which may provide a better opportunity to observe either detrimental or beneficial effects of OCs on CF disease. We observed a drop of $\sim 1-2 \%$ pred in FEV1 per year in our cohorts, which is consistent with most recently published data [22], although reported rates of decline vary (see later). Importantly, the decline in lung function in females using and not using OCs was similar. In addition, subgroup analysis in younger $(<23 \mathrm{yrs})$ and older $(\geqslant 23 \mathrm{yrs})$ females did not indicate an effect of OC use on disease severity.

Consistent with data collated from other registries [22], we have shown here that lung function in females with CF aged $<23$ yrs declines more rapidly than in older females. The reasons for the steeper decline in FEV1 from early adolescence to early adulthood are poorly understood, but we show here that the younger population also has a lower BMI and an increased need for i.v. antibiotics. Importantly, we have shown that OC use does not alter the decline in lung function in this younger cohort.

The reported rate of decline in lung function (FEV1 \% pred) varies from study to study ([23] and references therein), and also appears to be birth-cohort dependent. Reported values range from $0.6 \%$ to $4 \%$ decline in FEV1 per year. In addition, several studies have shown that anti-inflammatory drugs such as prednisone and ibuprofen improve, or slow, the decline of lung function over a 2-4-yr study period [24-26]. It is, therefore, reasonable to expect a significant positive or negative effect of OC use to become apparent over a 3-5-yr study period. Milder detrimental or protective effects of OCs would probably become more obvious the longer the period of continuous use of OCs. This, however, needs to be balanced against the inverse relationship between length of OC use and available patient numbers. For example, only $\sim 10 \%(n=31)$ of $C F$ females in our database had taken OCs for a continuous period of 6 or 7 yrs. The inter-patient cohort comparison had sufficient statistical power to detect a $\sim 10 \%$ difference in the absolute value of FEV1 $\%$ pred in females exposed and not exposed to OCs. Smaller effects of OCs on lung function may have been missed, but are unlikely to have been of clinical significance. For the interpatient analysis, we matched females for year of birth and age to minimise birth cohort- and age-related disease severity effects. Furthermore, we assessed the effects of OC use on three key markers of CF disease severity; lung function as measured by FEV1 \% pred, BMI and i.v. antibiotic use. To assess if age at time of OC use is an important variable, we also performed a subgroup analysis in younger and older females, but again OCs did not appear to have an effect.

In addition to inter-patient analysis we also performed an intrapatient analysis, by comparing periods of OC use to subsequent periods of no OC use in the same patient and vice versa. Intra-patient analysis is less likely to be affected by confounding factors such as genetics, environment or medication. As noted above, we had to balance the need for prolonged use of OCs 


\begin{tabular}{|c|c|c|c|c|c|c|}
\hline $\begin{array}{ll}\text { TABLE } 3 & \text { Intra-sub } \\
\text { period) }\end{array}$ & $\begin{array}{l}\text { omparison of di } \\
\text { o OC use ("not }\end{array}$ & $\begin{array}{l}\text { e severity during a } \\
\text { sed" period) in fen }\end{array}$ & $\begin{array}{l}\text { nt } 3-y r \text { pe } \\
\text { with cyst }\end{array}$ & $\begin{array}{l}\text { ds of oral contrac } \\
\text { fibrosis }\end{array}$ & eptive (OC) use & exposed" \\
\hline \multirow[t]{2}{*}{ Parameter } & \multicolumn{3}{|c|}{ First 3-yr period } & \multicolumn{3}{|c|}{ Second 3-yr period } \\
\hline & Exposed period & Not exposed period & p-value ${ }^{\#}$ & Not exposed period & Exposed period & p-value ${ }^{\#}$ \\
\hline $\begin{array}{l}\Delta \text { FEV }_{1} \% \text { pred } \\
\Delta \text { BMI } \mathrm{kq} \cdot \mathrm{m}^{-2}\end{array}$ & $-0.40(-22.4-7.55)^{\circ}$ & $-2.00(-16.70-4.85)^{\bullet}$ & 0.265 & $-1.83(-15.50-6.60)^{5}$ & $-2.35(-9.30-6.65)^{\S}$ & 0.426 \\
\hline
\end{tabular}

against available patient numbers and, therefore, restricted the intra-patient analysis to 3-yr periods of OC/no OC use.

The lack of effect in our study is in contrast to previous studies in asthma where oestrogens have been shown to affect disease severity in female asthmatics. During low-oestrogen phases of the menstrual cycle hospitalisation and exacerbation rates appear to be increased and lung function reduced [27-29]. In addition, OC use has reduced exacerbation rates and improved lung function in some asthmatic females [17].

Similar to other retrospective studies, our study has a number of limitations. 1) We have no information about the type of OC used. OCs have been available in the UK since 1960, with the original pill containing oestrogen and progesterone [30]. However, more recently, progesterone-only "mini pills" have become widely available, although combination pills are still in use. In vitro studies have shown that progesterone can also impair chloride ion transport [31] or increase CFTR expression [14]. 2) Females with CF routinely require antibiotics and it has been well described that antibiotics can impair the effectiveness of OCs by reducing circulating oestradiol levels [32]. We do not have pharmacokinetic data assessing hormone concentrations in our patient population and, therefore, cannot completely exclude the possibility that circulating oestrogen and/or progesterone levels may have been affected by simultaneous antibiotic intake and may have been too low to affect ion transport properties. However, to the best of our knowledge, the rate of unplanned pregnancies in females with CF is not higher than in the general population, which implies that circulating hormone levels are not significantly reduced by the antibiotics. 3) We have no information as to why females were using OCs. Avoidance of pregnancy is the most likely explanation, but prevention of irregular menstruation may also be a reason. Females may be using OCs because they are very well and want to avoid pregnancy or they make take OCs because their health has declined and would, therefore, not be able to cope with pregnancy. It is conceivable that these scenarios may have led to significant differences in disease severity between the two cohorts. However, we assessed stratification of the cohorts at the start of the study period and showed that there were no differences in disease severity as measured by FEV1 \% pred, BMI and use of i.v. antibiotics. 4) We carefully matched cohort subjects for year of birth and time period and era of OC use or non-use, but did not match for other variables that may influence disease, such as age of first $P$. aeruginosa isolate and treatments during the study period, which would have further reduced numbers. 5) Missing data or the lack of appropriate control subjects can be a problem in retrospective studies; we had to exclude $15 \%$ of females because we did not have any information related to OC use or because appropriate controls could not be identified. However, we think it improbable that any missing or erroneous information on the database would be systematically related to our study hypothesis and, thus, is unlikely to have produced any bias in our findings. 6) The study is based on data collected in a single centre and reproducibility of the results should ideally be assessed nationwide. However, current UK and US registry data do not record this. We are currently assessing whether questions related to types of OC used and reasons for OC use could be usefully added to the annual review questionnaire, to allow us to collect longitudinal data, which may help to reinforce the conclusions drawn in this study.

In summary, OC use did not affect FEV1 \% pred, BMI or the need for i.v. antibiotics. These findings, therefore, suggest that the use of OCs does not affect CF disease severity.

\section{SUPPORT STATEMENT}

The work was funded, in part, by the Cystic Fibrosis Trust and the Dr Benjamin Angel Senior Fellowship (U. Griesenbach). The project was supported by the National Institute for Health Research Respiratory Disease Biomedical Research Unit at the Royal Brompton and Harefield NHS Foundation Trust and Imperial College London, London, UK.

\section{STATEMENT OF INTEREST}

None declared.

\section{ACKNOWLEDGEMENTS}

We thank M. Balkin (Royal Brompton and Harefield NHS Foundation Trust, London, UK) for help with the Dendrite database and L. Hellings (Imperial College London, London, UK) for help with preparing the manuscript.

\section{REFERENCES}

1 Kreindler JL. Cystic fibrosis: exploiting its genetic basis in the hunt for new therapies. Pharmacol Ther 2010; 125: 219-229.

2 Dorfman R, Zielenski J. Genotype-phenotype correlations in cystic fibrosis. In: Bush A, Alton EWFW, Davies JC, et al., eds. Cystic Fibrosis in the 21st Century. London, Karger, 2009; pp. 61-68. 
3 Dodge JA, Lewis PA, Stanton M, et al. Cystic fibrosis mortality and survival in the UK: 1947-2003. Eur Respir J 2007; 29: 522-526.

4 Hodson ME, Simmonds NJ, Warwick WJ, et al. An international/ multicentre report on patients with cystic fibrosis (CF) over the age of 40 years. J Cyst Fibros 2008; 7: 537-542.

5 Rosenfeld M, Davis R, FitzSimmons S, et al. Gender gap in cystic fibrosis mortality. Am J Epidemiol 1997; 145: 794-803.

6 Corey M, Edwards L, Levison H, et al. Longitudinal analysis of pulmonary function decline in patients with cystic fibrosis. J Pediatr 1997; 131: 809-814.

7 Demko CA, Byard PJ, Davis PB. Gender differences in cystic fibrosis: Pseudomonas aeruginosa infection. J Clin Epidemiol 1995; 48: 1041-1049.

8 Verma N, Bush A, Buchdahl R. Is there still a gender gap in cystic fibrosis? Chest 2005; 128: 2824-2834.

9 Sweezey NB, Smith D, Corey M, et al. Amiloride-insensitive nasal potential difference varies with the menstrual cycle in cystic fibrosis. Pediatr Pulmonol 2007; 42: 519-524.

10 Singh AK, Schultz BD, Katzenellenbogen JA, et al. Estrogen inhibition of cystic fibrosis transmembrane conductance regulatormediated chloride secretion. J Pharmacol Exp Ther 2000; 295: 195-204.

11 Coakley RD, Sun $\mathrm{H}$, Clunes LA, et al. 17ß-estradiol inhibits $\mathrm{Ca}^{2+}$ dependent homeostasis of airway surface liquid volume in human cystic fibrosis airway epithelia. J Clin Invest 2008; 118: 4025-4035.

12 Nobuzane T, Tashiro S, Kudo Y. Morphologic effects of epithelial ion channels on the mouse uterus: differences between raloxifene analog (LY117018) and estradiol treatments. Am J Obstet Gynecol 2008; 199: 363-366.

13 Rowlands DK, Tsang LL, Cui YG, et al. Upregulation of cystic fibrosis transmembrane conductance regulator expression by oestrogen and Bak Foong Pill in mouse uteri. Cell Biol Int 2001; 25: 1033-1035.

14 Laube M, Kuppers E, Thome UH. Modulation of sodium transport in alveolar epithelial cells by estradiol and progesterone. Pediatr Res 2011; 69: 200-205.

15 Fanelli T, Cardone RA, Favia $\mathrm{M}$, et al. $\beta$-oestradiol rescues $\triangle$ F508CFTR functional expression in human cystic fibrosis airway CFBE41o- cells through the up-regulation of NHERF1. Biol Cell 2008; 100: 399-412.

16 Chotirmall SH, Greene CM, Oglesby IK, et al. $17 \beta$-estradiol inhibits IL-8 in cystic fibrosis by up-regulating secretory leucoprotease inhibitor. Am J Respir Crit Care Med 2010; 182: 62-72.
17 Haggerty CL, Ness RB, Kelsey S, et al. The impact of estrogen and progesterone on asthma. Ann Allergy Asthma Immunol 2003; 90: 284-291.

18 Johannesson M, Ludviksdottir D, Janson C. Lung function changes in relation to menstrual cycle in females with cystic fibrosis. Respir Med 2000; 94: 1043-1046.

19 Fitzpatrick SB, Stokes DC, Rosenstein BJ, et al. Use of oral contraceptives in women with cystic fibrosis. Chest 1984; 86: 863-867.

20 Plant BJ, Goss CH, Tonelli MR, et al. Contraceptive practices in women with cystic fibrosis. J Cyst Fibros 2008; 7: 412-414.

21 Conway SP, Morton AM, Oldroyd B, et al. Osteoporosis and osteopenia in adults and adolescents with cystic fibrosis: prevalence and associated factors. Thorax 2000; 55: 798-804.

22 Liou TG, Elkin EP, Pasta DJ, et al. Year-to-year changes in lung function in individuals with cystic fibrosis. J Cyst Fibros 2010; 9: 250-256.

23 Que C, Cullinan P, Geddes D. Improving rate of decline of FEV1 in young adults with cystic fibrosis. Thorax 2006; 61: 155-157.

24 Eigen H, Rosenstein BJ, FitzSimmons S, et al. A multicenter study of alternate-day prednisone therapy in patients with cystic fibrosis. Cystic Fibrosis Foundation Prednisone Trial Group. J Pediatr 1995; 126: 515-523.

25 Konstan MW, Byard PJ, Hoppel CL, et al. Effect of high-dose ibuprofen in patients with cystic fibrosis. N Engl J Med 1995; 332: 848-854.

26 Lands LC, Milner R, Cantin AM, et al. High-dose ibuprofen in cystic fibrosis: Canadian safety and effectiveness trial. J Pediatr 2007; 151: 249-254.

27 Eliasson O, Scherzer HH. Recurrent respiratory failure in premenstrual asthma. Conn Med 1984; 48: 777-778.

28 Eliasson O, Scherzer HH, DeGraff AC Jr. Morbidity in asthma in relation to the menstrual cycle. J Allergy Clin Immunol 1986; 77: 87-94.

29 Gibbs CJ, Coutts II, Lock R, et al. Premenstrual exacerbation of asthma. Thorax 1984; 39: 833-836.

30 Junod SW, Marks L. Women's trials: the approval of the first oral contraceptive pill in the United States and Great Britain. J Hist Med Allied Sci 2002; 57: 117-160.

31 Sweezey NB, Gauthier C, Gagnon S, et al. Progesterone and estradiol inhibit CFTR-mediated ion transport by pancreatic epithelial cells. Am J Physiol 1996; 271: G747-G754.

32 Dickinson BD, Altman RD, Nielsen NH, et al. Drug interactions between oral contraceptives and antibiotics. Obstet Gynecol 2001; 98: 853-860. 